\title{
Dielectric relaxation in dipolar fluids. Generalized mode approach
}

\author{
I.P.Omelyan, I.M.Mryglod, M.V.Tokarchuk \\ Institute for Condensed Matter Physics of the National Academy of \\ Sciences of Ukraine, 1 Svientsitskii St., UA-290011 Lviv-11, Ukraine
}

Received November 28, 1997

The concept of generalized collective modes, recently proposed for the investigation of simple fluids, is now applied to describe processes of dielectric relaxation in dipolar systems. The approach presented here is an extension of the dipole-density formalism to arbitrary numbers of dynamical variables and values of wavelengths. Generalized dipolar mode spectra of a Stockmayer fluid are evaluated over a wide scale of wavelengths up to the five-variable approximation. The wavevector- and frequency-dependent dielectric permittivity and dipole-moment time autocorrelation functions are calculated on the basis of analytical expressions using the dipolar modes. The obtained results are compared with those achieved in lower-order approximations and with molecular dynamics data. It is shown that the fivevariable description quantitatively reproduces the entire frequency dependence of the dielectric constant at arbitrary wavenumbers.

Key words: collective modes; dielectric properties; extended hydrodynamics

PACS: $67.55 . J d ; 77.22 .-d ; 47.65$

\section{Introduction}

Processes of dielectric relaxation in polar fluids have been intensively studied in theory [1-7], computer simulation [8-15] and pure experiment [16-18]. Nevertheless, there is still lack of agreement between the theoretically predicted and experimental results. Moreover, some main problems remain unsolved even in the simplest case when the fluid is treated as a system of point dipoles. Among various theoretical schemes, enabling us to describe the processes of dielectric relaxation in dipolar fluids, it is necessary to point out two consequent approaches: the dipoledensity formalism of Madden and Kivelson [2] and the extended hydrodynamic description popularized by Bagchi and Chandra [4].

The usual Navier-Stokes hydrodynamics [19], being valid in the infinite-wavelength limit and large times, cannot be used at molecular length scales. In the extended hydrodynamic description, the microscopic operators of the number density 
$\hat{n}(\boldsymbol{r}, \boldsymbol{\Theta})=\sum_{i=1}^{N} \delta\left(\boldsymbol{r}-\boldsymbol{r}_{i}\right) \delta\left(\boldsymbol{\Theta}-\boldsymbol{\Theta}_{i}\right)$, spatial $\hat{\boldsymbol{P}}(\boldsymbol{r}, \Theta)=\sum_{i=1}^{N} m \boldsymbol{v}_{i} \delta\left(\boldsymbol{r}-\boldsymbol{r}_{i}\right) \delta\left(\boldsymbol{\Theta}-\mathbf{\Theta}_{i}\right)$ and angular $\hat{\boldsymbol{L}}(\boldsymbol{r}, \boldsymbol{\Theta})=\sum_{i=1}^{N} J \boldsymbol{w}_{i} \delta\left(\boldsymbol{r}-\boldsymbol{r}_{i}\right) \delta\left(\boldsymbol{\Theta}-\boldsymbol{\Theta}_{i}\right)$ momenta densities are considered as basic dynamical quantities [6]. These quantities satisfy modified equations which take into account processes with short and intermediate time scales as well. In their theory, fluctuations of the total energy density $\hat{e}(\boldsymbol{r}, \boldsymbol{\Theta})=\sum_{i=1}^{N} e_{i} \delta(\boldsymbol{r}-$ $\left.\boldsymbol{r}_{i}\right) \delta\left(\boldsymbol{\Theta}-\boldsymbol{\Theta}_{i}\right)$, where $e_{i}=\frac{1}{2}\left(m \boldsymbol{v}_{i}^{2}+J \boldsymbol{w}_{i}^{2}+\sum_{j(j \neq i)}^{N} \varphi_{i j}\right)$, are not taken into account because of the complexities of hydrodynamic equations. Vector $\boldsymbol{\Theta}_{i}$ characterizes orientations of molecule $i$ and, in the case of rigid nonpolarizable molecules, it can be associated with the unit vector directed along the particle's dipole moment, i.e., $\boldsymbol{\Theta}_{i}=\boldsymbol{\mu}_{i} / \mu$. Then, the dipole-moment fluctuations can be reproduced on the basis of number-density correlations using the relation $\hat{\boldsymbol{M}}(\boldsymbol{r}, t)=\mu\langle\boldsymbol{\Theta} \hat{n}(\boldsymbol{r}, \boldsymbol{\Theta}, t)\rangle_{\boldsymbol{\Theta}}$ where the averaging is performed over orientations. In such a way the dynamical polarization in a dense dipolar fluid can be investigated, provided reasonable forms of the dissipative kernels are available. Despite the fact that such an approach allows us, in principle, to describe the processes of dielectric relaxation in a dipolar fluid and connect them directly with thermodynamic and hydrodynamic properties, it is rather sophisticated and impractical in application. The main results are yet to be obtained here.

A somewhat different approach to describing the generalized hydrodynamics of dipolar systems has been recently proposed in [20]. The main idea of this approach consists in the following. When vector $\boldsymbol{M}(\boldsymbol{k})$ of the dipole density is already included into the consideration as an orientational variable, it is no longer necessary to deal with the orientational dependence $\hat{\boldsymbol{Q}}(\boldsymbol{r}, \boldsymbol{\Theta}) \equiv\{\hat{n}, \hat{\boldsymbol{P}}, \hat{e}, \hat{\boldsymbol{L}}\}$ of hydrodynamic variables. Then the basic set can be cast in the usual molecular form $\hat{\boldsymbol{Q}}(\boldsymbol{k})=\int_{V}\langle\hat{\boldsymbol{Q}}(\boldsymbol{r}, \boldsymbol{\Theta})\rangle_{\Theta} \mathrm{e}^{-\mathrm{i} \boldsymbol{k} \cdot \boldsymbol{r}} \mathrm{d} \boldsymbol{r} \equiv\{n(\boldsymbol{k}), \boldsymbol{P}(\boldsymbol{k}), e(\boldsymbol{k}), \boldsymbol{L}(\boldsymbol{k})\}$, where $n(\boldsymbol{k})=$ $\sum_{i=1}^{N} \mathrm{e}^{-\mathrm{i} \boldsymbol{k} \cdot \boldsymbol{r}_{i}}, \boldsymbol{P}(\boldsymbol{k})=\sum_{i=1}^{N} m \boldsymbol{v}_{i} \mathrm{e}^{-\mathrm{i} \boldsymbol{k} \cdot \boldsymbol{r}_{i}}, e(\boldsymbol{k})=\sum_{i=1}^{N}\left\langle e_{i}\right\rangle_{\boldsymbol{\Theta}_{i}} \mathrm{e}^{-\mathrm{i} \boldsymbol{k} \cdot \boldsymbol{r}_{i}}$ and $\boldsymbol{L}(\boldsymbol{k})=\sum_{i=1}^{N}$ $J \boldsymbol{w}_{i} \mathrm{e}^{-i \boldsymbol{k} \cdot \boldsymbol{r}_{i}}$. The advantage of such a representation lies in the evident simplification of the corresponding hydrodynamic equations. Moreover, owing to conservation laws of the total number of particles, momenta and energy, the basic set $\hat{\boldsymbol{Q}}(\boldsymbol{k})$ can be considered as a set of indeed slow variables. For instance, time derivatives of $n(\boldsymbol{k}), \boldsymbol{P}(\boldsymbol{k})$ and $e(\boldsymbol{k})$ vanish when $k \rightarrow 0$. Finally, for isotropic and spatially homogeneous systems, the dipole density $\boldsymbol{M}(\boldsymbol{k})$ does not correlate at equilibrium with the basic hydrodynamic quantities in the static limit, i.e., $\langle\boldsymbol{M}(\boldsymbol{k}) \cdot \hat{\boldsymbol{Q}}(\boldsymbol{k})\rangle=0$.

For this reason, the dipole-density and hydrodynamic fluctuations can be considered separately from each other as this has been assumed implicitly in the dipole-density formalism of Madden and Kivelson [2]. The generalized collective modes related to dipole-density fluctuations will be called dipolar modes in order to distinguish them from the hydrodynamic modes, concerning fluctuations of hydrodynamic quantities. At the same time, the generalized hydrodynamic modes of a dipolar system can be studied within the same scheme as in the case of simple fluids [21-24]. It is also worth remarking that the correlations between the dipolemoment density and hydrodynamic quantities are not completely independent. They, being absent at the basic level, appear, however, at higher-orders of the 
description. This leads to additional non-Markovian effects, when working within the dipole-density formalism. Nevertheless, these effects are taken into account in an effective way, including, besides the basic quantity $\boldsymbol{M}(\boldsymbol{k})$, its higher-order components as well. The hydrodynamic correlations can be included in the same way, too. Time constants appearing in the dissipative kernel are usually considered, as adjustable parameters. It is worth stressing that the previous applications of this theory were restricted to the long-wavelength regime only [12].

Recently, the concept of generalized collective modes, used earlier for the investigation of nonequilibrium properties of simple fluids [21-25], has been applied to dipolar systems and actual computations have been performed in the whole wavevector range within the three-mode description [26]. In particular, it has been concluded that the three-variable prescription is sufficient to predict quantitatively the frequency dependence of dielectric quantities for a Stockmayer fluid. But such a conclusion was based on the calculations carried out with the help of a fitting procedure, because a higher-order static correlation function was not known. For this reason, it is not obvious that the described above pattern takes place indeed.

In the present paper, the generalized dipolar mode spectra of a Stockmayer fluid are evaluated over a wide scale of wavelengths up to the five-variable description without involving any adjustable parameters. Within the framework of the proposed approach, the frequency dependence of dielectric quantities is determined by extended continued fractions to which the Markovian approximation is applied. As a result, by using the dipolar modes it is shown that the threevariable theory reproduces qualitatively the wavevector- and frequency-dependent dielectric constant and only beginning from the five-order description one can talk about a quantitative reproduction over the whole range of varying wavenumbers and frequencies. Moreover, we demonstrate that within the same approximation all times constants of memory kernels can be expressed in terms of static correlation functions, so that dynamic properties of the system are obtained using static fluctuations exclusively.

\section{General theoretical framework}

\subsection{Basic definitions}

We shall consider a dipolar fluid of $N$ identical molecules enclosed in volume $V$. Let

$$
\boldsymbol{M}(\boldsymbol{k}, t)=\sum_{i=1}^{N} \boldsymbol{\mu}_{i}(t) \mathrm{e}^{-\mathrm{i} \boldsymbol{k} \cdot \boldsymbol{r}_{i}(t)}
$$

be the primary dynamical variable whose correlation function is of direct interest. The collective variable (1) presents, in fact, the spatial Fourier transform $\int_{V} \hat{\boldsymbol{M}}(\boldsymbol{r}, t) \mathrm{e}^{-\mathrm{i} \boldsymbol{k} \cdot \boldsymbol{r}} \mathrm{d} \boldsymbol{r}$ of the microscopic operator of dipole-moment density $\hat{\boldsymbol{M}}(\boldsymbol{r}, t)=\sum_{i=1}^{N} \boldsymbol{\mu}_{i}(t) \delta\left(\boldsymbol{r}-\boldsymbol{r}_{i}(t)\right)$ where $\boldsymbol{r}_{i}(t)$ and $\boldsymbol{\mu}_{i}(t)$ denote the dipole moment and position, respectively, of molecule $i$ at time $t$. This dynamical variable satisfies 
the equation of motion $\mathrm{d} \boldsymbol{M}(\boldsymbol{k}, t) / \mathrm{d} t=L \boldsymbol{M}(\boldsymbol{k}, t)$, where

$$
L=\sum_{i=1}^{N}\left(\boldsymbol{v}_{i} \cdot \frac{\partial}{\partial \boldsymbol{r}_{i}}+\boldsymbol{w}_{i} \times \boldsymbol{\mu}_{i} \cdot \frac{\partial}{\partial \boldsymbol{\mu}_{i}}\right)-\sum_{i \neq j}^{N}\left(\frac{1}{m} \frac{\partial \varphi_{i j}}{\partial \boldsymbol{r}_{i}} \cdot \frac{\partial}{\partial \boldsymbol{v}_{i}}+\frac{1}{J} \boldsymbol{\mu}_{i} \times \frac{\partial \varphi_{i j}}{\partial \boldsymbol{\mu}_{i}} \cdot \frac{\partial}{\partial \boldsymbol{w}_{i}}\right)
$$

is the Liouville operator of the system, $\varphi_{i j}$ denotes an intermolecular potential, $\boldsymbol{v}_{i}$ and $\boldsymbol{w}_{i}$ are the translational and rotational velocities, respectively, of molecule $i$ with mass $m$ and moment of inertia $J$. The formal solution of the equation of motion is $\boldsymbol{M}(\boldsymbol{k}, t)=\mathrm{e}^{L t} \boldsymbol{M}(\boldsymbol{k}, 0) \equiv \mathrm{e}^{L t} \boldsymbol{M}(\boldsymbol{k})$.

Let us consider the extended set of variables

$$
\left\{\boldsymbol{M}(\boldsymbol{k}), L \boldsymbol{M}(\boldsymbol{k}), L^{2} \boldsymbol{M}(\boldsymbol{k}), \ldots, L^{S-1} \boldsymbol{M}(\boldsymbol{k})\right\} \equiv\left\{L^{\alpha-1} \boldsymbol{M}(\boldsymbol{k})\right\}, \quad \alpha=1, \ldots, S
$$

which includes, besides the basic dynamical quantity $\boldsymbol{M}(\boldsymbol{k})$, its time derivatives at $t=0$ up to the $(S-1)$-th order. It is necessary to note that due to isotropy of the system, longitudinal and transverse fluctuations are completely independent of one another and can be studied separately. Therefore, set (3) is formed by either longitudinal, $\boldsymbol{M}^{\mathrm{L}}(\boldsymbol{k})$, or transverse, $\boldsymbol{M}^{\mathrm{T}}(\boldsymbol{k})$, components of $\boldsymbol{M}(\boldsymbol{k})$. For the convenience of further presentation we transform the extended set to the orthogonalized form

$$
\mathscr{M}(\boldsymbol{k})=\left\{\mathscr{M}_{1}(\boldsymbol{k}), \mathscr{M}_{2}(\boldsymbol{k}), \mathscr{M}_{3}(\boldsymbol{k}), \ldots, \mathscr{M}_{S}(\boldsymbol{k})\right\},
$$

where $\mathscr{M}_{1}(\boldsymbol{k})=\boldsymbol{M}(\boldsymbol{k}), \mathscr{M}_{2}(\boldsymbol{k})=\left(1-\mathscr{P}_{1}\right) L \boldsymbol{M}(\boldsymbol{k}), \ldots, \mathscr{M}_{S}(\boldsymbol{k})=\left(1-\mathscr{P}_{S-1}\right) L^{S-1}$ $\boldsymbol{M}(\boldsymbol{k})$ and $\mathscr{P}_{\alpha} \ldots=\sum_{\beta=1}^{\alpha}\left\langle\ldots \cdot \mathscr{M}_{\beta}(-\boldsymbol{k})\right\rangle\left\langle\mathscr{M}_{\beta}(\boldsymbol{k}) \cdot \mathscr{M}_{\beta}(-\boldsymbol{k})\right\rangle^{-1} \mathscr{M}_{\beta}(\boldsymbol{k})$ denotes the Mori-like projection operator $[27,28]$. The orthogonalized variables (4) constitute the matrix of time correlation functions (TCFs)

$$
f_{\alpha \beta}(k, t)=\frac{\left\langle\mathscr{M}_{\alpha}(\boldsymbol{k}) \cdot \mathrm{e}^{L t} \mathscr{M}_{\beta}(-\boldsymbol{k})\right\rangle}{\{2\} N \mu^{2}} \equiv \mathbf{F}(k, t)
$$

which is diagonal in the static limit $t \rightarrow 0$, i.e., $f_{\alpha \beta}(k)=\delta_{\alpha \beta} f_{\alpha \alpha}(k)$ where \langle\rangle denotes the equilibrium average, $\mu$ designates the permanent magnitude of the molecule's dipole moment and the multiplier $\{2\}$ is included in the case of transverse fluctuations only.

According to the construction of the orthogonalized set, the functions $f_{\alpha \alpha}(k)$ can be found in terms of static correlation functions (SCFs) corresponding to the initial set $(3), g_{\alpha \beta}(k)=\left\langle L^{\alpha-1} \boldsymbol{M}(\boldsymbol{k}) \cdot L^{\beta-1} \boldsymbol{M}(-\boldsymbol{k})\right\rangle /\{2\} N \mu^{2}$, by the iterations

$$
\xi_{\alpha \beta}(k)=g_{\alpha \beta}(k)-\sum_{\gamma=1}^{\beta-1} \frac{\xi_{\alpha \gamma}(k) \xi_{\beta \gamma}(k)}{f_{\gamma \gamma}(k)}, \quad f_{\alpha \alpha}(k)=g_{\alpha \alpha}(k)-\sum_{\gamma=1}^{\alpha-1} \frac{\xi_{\alpha \gamma}^{2}(k)}{f_{\gamma \gamma}(k)}
$$

with $f_{11}(k)=g_{11}(k)$ and $\alpha=2,3, \ldots, S ; \beta=1,2, \ldots, \alpha-1$, where it is understood that the sum in the right-hand side of the first equality of (6) must be omitted at $\beta=1$ and $\xi_{\alpha \beta}(k)=\left\langle L^{\alpha-1} \boldsymbol{M}(\boldsymbol{k}) \cdot \mathscr{M}_{\beta}(-\boldsymbol{k})\right\rangle /\{2\} N \mu^{2}$ denotes an auxiliary matrix. 
The orthogonalized procedure can be simplified significantly taking into account that the basic function $g(k, t) \equiv f_{11}(k, t)$ is even with respect to time. Then one obtains that $g_{\alpha \beta}(k)$ (as well as $\xi_{\alpha \beta}(k)$ ) are equal to zero if $\alpha+\beta$ is an odd number, whereas nonzero elements can be expressed via their diagonal ones as $g_{\alpha \beta}(k)=$ $(-1)^{\frac{|\alpha-\beta|}{2}} g_{\gamma \gamma}(k)$, where $\gamma=(\alpha+\beta) / 2$.

The processes of dynamical polarization in the system can be described by TCFs (5). In particular, the longitudinal $\varepsilon_{\mathrm{L}}(k, \omega)$ and transverse $\varepsilon_{\mathrm{T}}(k, \omega)$ components of the wavevector- and frequency-dependent dielectric permittivity are expressed via the first element $g(k, t)$ of the $S \times S$ square matrix $\mathbf{F}(k, t)$ as [15]:

$$
\frac{\varepsilon_{\mathrm{L}}(k, \omega)-1}{9 y \varepsilon_{\mathrm{L}}(k, \omega)}=g^{\mathrm{L}}(k)-\mathrm{i} \omega g^{\mathrm{L}}(k, \omega), \quad \frac{\varepsilon_{\mathrm{T}}(k, \omega)-1}{9 y}=g^{\mathrm{T}}(k)-\mathrm{i} \omega g^{\mathrm{T}}(k, \omega),
$$

where $y=4 \pi N \mu^{2} / 9 V k_{\mathrm{B}} T$ and $k_{\mathrm{B}}, T$ are the Boltzmann constant and temperature of the system, respectively, $g(k, \omega)=\int_{0}^{\infty} g(k, t) \mathrm{e}^{-\mathrm{i} \omega t} \mathrm{~d} t \equiv \mathscr{L}_{\mathrm{i} \omega}(g(k, t))$ and $\mathscr{L}_{\mathrm{i} \omega}$ designates the Laplace transform.

\subsection{Extended continued fractions}

Using the memory function formalism, the matrix equation for equilibrium TCFs can be written as follows [21]:

$$
\frac{\partial}{\partial t} \mathbf{F}(k, t)-\boldsymbol{\Omega}(k) \mathbf{F}(k, t)+\int_{0}^{\infty} \boldsymbol{\Gamma}(k, \tau) \mathbf{F}(k, t-\tau) \mathrm{d} \tau=0
$$

In our case, due to the orthogonality of (4), the matrix of memory kernels $\boldsymbol{\Gamma}(k, \tau)$ has one nonzero element only, namely, $\Gamma_{S S}(k, \tau) \equiv \Gamma_{S}(k, \tau)$, where

$$
\Gamma_{S}(k, \tau)=\frac{\left\langle\left(\left(1-\mathscr{P}_{S}\right) \mathscr{M}_{S+1}(\boldsymbol{k}) \cdot \exp \left(\left(1-\mathscr{P}_{S}\right) L \tau\right)\left(1-\mathscr{P}_{S}\right) \mathscr{M}_{S+1}(-\boldsymbol{k})\right\rangle\right.}{\left\langle\mathscr{M}_{S}(\boldsymbol{k}) \cdot \mathscr{M}_{S}(-\boldsymbol{k})\right\rangle}
$$

and

$$
\boldsymbol{\Omega}(k)=\frac{\left\langle\mathscr{M}(\boldsymbol{k}) \cdot L \mathscr{M}^{+}(\boldsymbol{k})\right\rangle}{\left\langle\mathscr{M}(\boldsymbol{k}) \cdot \mathscr{M}^{+}(\boldsymbol{k})\right.}=\left(\begin{array}{cccccc}
0 & -1 & 0 & 0 & 0 & \ldots \\
\Omega_{2} & 0 & -1 & 0 & 0 & \ldots \\
0 & \Omega_{3} & 0 & -1 & 0 & \ldots \\
0 & 0 & \Omega_{4} & 0 & -1 & \ldots \\
0 & 0 & 0 & \Omega_{5} & 0 & \ldots \\
\ldots & \ldots & \ldots & \ldots & \ldots & \ldots
\end{array}\right)
$$

is the frequency matrix with the elements

$$
\Omega_{\alpha}(k)=\frac{\left\langle\mathscr{M}_{\alpha}(\boldsymbol{k}) \cdot \mathscr{M}_{\alpha}(-\boldsymbol{k})\right\rangle}{\left\langle\mathscr{M}_{\alpha-1}(\boldsymbol{k}) \cdot \mathscr{M}_{\alpha-1}(-\boldsymbol{k})\right\rangle} \equiv \frac{f_{\alpha \alpha}(k)}{f_{\alpha-1 \alpha-1}(k)} .
$$


In the Laplace representation, the integro-differential equation (8) converts into the algebraic one

$$
[i \omega \mathbf{I}-\boldsymbol{\Omega}(k)+\boldsymbol{\Gamma}(k, \omega)] \mathbf{F}(k, \omega)=\mathbf{F}(k),
$$

where $\mathbf{I}$ is the unit matrix and $\mathbf{F}(k)=\lim _{t \rightarrow 0} \mathbf{F}(k, t)$ denotes the matrix of SCFs. The equation (12) is now solved analytically and the result for the first element of $\mathbf{F}(k, \omega)$ can be cast as the $S$-th order continued fraction,

$$
g(k, \omega)=\frac{g(k)}{\mathrm{i} \omega+\frac{\Omega_{2}(k)}{\mathrm{i} \omega+\frac{\Omega_{3}(k)}{\mathrm{i} \omega+\ldots \frac{\Omega_{S}(k)}{\mathrm{i} \omega+\Gamma_{S}(k, \omega)}}}} .
$$

In view of (6), more explicit expressions for the elements (11) of the frequency matrix at $S \leqslant 5$ are

$$
\begin{aligned}
& \Omega_{2}(k)=\frac{g_{2}(k)}{g(k)}, \\
& \Omega_{3}(k)=\frac{g(k) g_{4}(k)-g_{2}^{2}(k)}{g(k) g_{2}(k)}, \\
& \Omega_{4}(k)=\frac{g(k)\left(g_{2}(k) g_{6}(k)-g_{4}^{2}(k)\right)}{g_{2}(k)\left(g(k) g_{4}(k)-g_{2}^{2}(k)\right)}, \\
& \Omega_{5}(k)=g_{2}(k)\left(g(k) g_{4}(k) g_{8}(k)+2 g_{2}(k) g_{4}(k) g_{6}(k)-g_{2}^{2}(k) g_{8}(k)-\right. \\
& \left.\quad g(k) g_{6}^{2}(k)-g_{4}^{3}(k)\right) /\left(g(k) g_{4}(k)-g_{2}^{2}(k)\right)\left(g_{2}(k) g_{6}(k)-g_{4}^{2}(k)\right),
\end{aligned}
$$

where

$$
g_{2 s}(k)=\frac{\left\langle L^{s} \boldsymbol{M}(\boldsymbol{k}) \cdot L^{s} \boldsymbol{M}(-\boldsymbol{k})\right\rangle}{\{2\} N \mu^{2}}
$$

is the static Kirkwood factor of the $2 s$-th order $(s=0,1, \ldots, S-1)$ and $g_{0}(k) \equiv$ $g(k)$.

It can be easily seen from (13) that the same function $g(k, \omega)$ is obtained within the $(S+1)$-th order continued fraction, too, if the memory functions obey the recurrent relation

$$
\Gamma_{S}(k, \omega)=\frac{\Omega_{S+1}(k)}{\mathrm{i} \omega+\Gamma_{S+1}(k, \omega)} .
$$

The solution (13) leads to exact results at arbitrary order $S$, provided the corresponding memory functions $\Gamma_{S}$ are precisely determined. However, such a prescription is rather a formal one, because the exact calculation (9) of the dissipative kernel constitutes, in general, an unresolvable problem. We shall now consider a question of how to perform this calculation approximately. 
Let

$$
\tau_{S}(k)=\min _{\alpha=1} \frac{\int_{0}^{\infty} t\left|f_{\alpha \alpha}(k, t)\right| \mathrm{d} t}{\int_{0}^{\infty}\left|f_{\alpha \alpha}(k, t)\right| \mathrm{d} t}, \quad \tau_{S+1}(k)=\max _{\alpha=S+1}^{\infty} \frac{\int_{0}^{\infty} t\left|f_{\alpha \alpha}(k, t)\right| \mathrm{d} t}{\int_{0}^{\infty}\left|f_{\alpha \alpha}(k, t)\right| \mathrm{d} t}
$$

be characteristic intervals of decaying in time of the autocorrelation functions from the $S$-order set and all the rest functions from higher-order sets, and $\gamma_{S}(k)=$ $\tau_{S+1}(k) / \tau_{S}(k)$ be their ratio. We assume in advance that there exist dynamical processes in the system, corresponding to essentially different scales of time and, therefore, beginning from some number $S$, the ratio $\gamma_{S}$ must become sufficiently small, i.e., $\gamma_{S}(k) \ll 1$ at arbitrary wavevectors. This assumption is justified provided that the $S$-order set (4) forms an almost complete slow set of dynamical variables. It is obvious that in this case the memory kernel (9), which is built on projected higher-order variables, decays in time faster than TCFs (5), i.e.,

$$
\int_{0}^{\infty} \boldsymbol{\Gamma}(k, \tau) \mathbf{F}(k, t-\tau) \mathrm{d} \tau \approx \int_{0}^{\infty} \boldsymbol{\Gamma}(k, \tau) \mathrm{d} \tau \mathbf{F}(k, t) \equiv \boldsymbol{\Gamma}(k) \mathbf{F}(k, t) .
$$

Thus, on characteristic time scales of varying TCFs, the dissipative kernel can be considered as a $\delta$-function in time space, $\Gamma_{S}(k, \tau) \approx \Gamma_{S}(k) \delta(\tau)$, with the weight $\Gamma_{S}(k) \equiv \Gamma_{S}(k, \omega=0)$. In the frequency representation the relation (18) takes the form:

$$
\Gamma_{S}(k, \omega) \approx \Gamma_{S}(k)
$$

that represents the well-known Markovian approximation [2, 4].

The memory kernel $\Gamma_{S}(k)$ can be found in terms of elements (11) of the frequency matrix and the basic wavevector-depended correlation time

$$
\tau_{\text {cor }}(k)=\lim _{\omega \rightarrow 0} \frac{g(k, \omega)}{g(k)}=\int_{0}^{\infty} \frac{g(k, t)}{g(k)} \mathrm{d} t
$$

putting $\omega \rightarrow 0$ in the $S$-order continued fraction (13). Then we obtain at different orders of the description the following result: $\Gamma_{1}(k)=1 / \tau_{\text {cor }}(k), \Gamma_{2}(k)=$ $\tau_{\text {cor }}(k) \Omega_{2}(k)$ and $(n=2,3, \ldots)$

$$
\Gamma_{2 n-1}(k)=\frac{1}{\tau_{\text {cor }}} \prod_{l=1}^{n-1} \frac{\Omega_{2(n-l)+1}}{\Omega_{2(n-l)}}, \quad \Gamma_{2 n}(k)=\tau_{\text {cor }} \Omega_{2} \prod_{l=1}^{n-1} \frac{\Omega_{2(n-l)+2}}{\Omega_{2(n-l)+1}} .
$$

It is worth mentioning that the frequency independence of the memory kernel in the $S$-order description does not concern the memory functions of lower orders which depend on frequency according to the recurrent relation (16).

And now we consider a more general formulation of the Markovian approximation in higher-order descriptions. Namely, as far as the ratio $\gamma_{S}(k)$ is small enough 
at a given value of $S$, it will remain small at higher orders, too, or will even decrease with increasing $S$. If this statement indeed takes place, then not only the frequency dependence of $\Gamma_{S}(k, \omega)$ can be neglected, but also the values of $\Gamma_{S}(k)$ will begin to be almost independent from the order $S$ of the description. Then we can write that $\Gamma_{S+1}(k) \approx \Gamma_{S}(k)$ at sufficiently great values of $S$ and the basic correlation time (20) can be excluded from our consideration. Taking into account the explicit relations $(21)$ and letting $\Gamma_{1}(k)=\Gamma_{2}(k)$ and $\Gamma_{2}(k)=\Gamma_{3}(k), \ldots, \Gamma_{2 n-1}(k)=\Gamma_{2 n}(k)$ and $\Gamma_{2 n}(k)=\Gamma_{2 n+1}(k)$, where $n=2,3, \ldots$, we obtain for the correlation time $\tau_{\text {cor }}^{(s)}(k)$ in the $s$-th approximation: $\tau_{\text {cor }}^{(1)}(k)=1 / \sqrt{\Omega_{2}(k)}, \tau_{\text {cor }}^{(2)}(k)=\sqrt{\Omega_{3}(k)} / \Omega_{2}(k)$, $\ldots$, and

$$
\begin{gathered}
\tau_{\mathrm{cor}}^{(2 n-1)}(k)=\frac{1}{\sqrt{\Omega_{2}}} \prod_{l=1}^{n-1} \frac{\Omega_{2(n-l)+1}}{\sqrt{\Omega_{2(n-l)} \Omega_{2(n-l)+2}}}, \\
\tau_{\text {cor }}^{(2 n)}(k)=\frac{\sqrt{\Omega_{3}}}{\Omega_{2}} \prod_{l=1}^{n-1} \frac{\sqrt{\Omega_{2(n-l)+1} \Omega_{2(n-l)+3}}}{\Omega_{2(n-l)+2}} .
\end{gathered}
$$

Finally, substituting values (22) into expressions (21) for memory functions within the same order of the approximation, we obtain

$$
\Gamma_{2 n-1}(k) \approx \sqrt{\Omega_{2}} \prod_{l=1}^{n-1} \sqrt{\frac{\Omega_{2(n-l)+2}}{\Omega_{2(n-l)}}}, \quad \Gamma_{2 n}(k) \approx \sqrt{\Omega_{3}} \prod_{l=1}^{n-1} \sqrt{\frac{\Omega_{2(n-l)+3}}{\Omega_{2(n-l)+1}}} .
$$

In such a way, in view of (13), (14) and (23) the frequency dependence (7) of the dielectric constant can be reproduced using static correlation functions (15) exclusively.

\subsection{Generalized collective modes}

In the preceding subsection it was shown how to obtain analytic results for dynamical quantities in the frequency representation. However, in the Markovian approximation, the equation (8) for TCFs can be solved analytically in time space as well. This equation can now be written as

$$
\frac{\partial}{\partial t} \mathbf{F}^{\mathrm{M}}(k, t)=-\mathbf{T}(k) \mathbf{F}^{\mathrm{M}}(k, t),
$$

where the generalized evolution operator $\mathbf{T}(k)=-\boldsymbol{\Omega}(k)+\boldsymbol{\Gamma}(k)$ is determined by explicit expressions (10), (11) and (21) (or (23)) for $\boldsymbol{\Omega}(k)$ and $\boldsymbol{\Gamma}(k)$, respectively, and $\mathbf{F}^{\mathrm{M}}$ indicates the matrix $\mathbf{F}$ of TCFs calculated in the Markovian approximation.

Let $X_{\beta \gamma}(k)$ be an eigenvector associated with the eigenvalue $Z_{\gamma}(k)$ of the $\mathbf{T}(k)$ matrix, i.e.,

$$
\sum_{\beta=1}^{S} T_{\alpha \beta}(k) X_{\beta \gamma}(k)=Z_{\gamma}(k) X_{\alpha \gamma}(k)
$$


where $\alpha, \gamma=1, \ldots, S$ and $T_{\alpha \beta}$ designate the elements of $\mathbf{T}$. Then the solution to differential equation (24) is of the form

$$
f_{\alpha \beta}^{\mathrm{M}}(k, t)=\sum_{\gamma=1}^{S} Q_{\gamma}^{\alpha \beta}(k) \mathrm{e}^{-Z_{\gamma}(k) t},
$$

i.e., each element of the $\mathbf{F}^{\mathrm{M}}$-matrix can be expressed as a sum of $S$ Lorentzians which are connected with the generalized collective modes $Z_{\gamma}(k)$. The amplitudes $Q_{\gamma}^{\alpha \beta}$ are defined in terms of eigenvectors uniquely, using the initial condition $\lim _{t \rightarrow 0} \mathbf{F}^{\mathrm{M}}(k, t)=\mathbf{F}(k)$. The result is

$$
Q_{\gamma}^{\alpha \beta}(k)=\sum_{l=1}^{S} X_{\alpha \gamma}(k) X_{\gamma l}^{-1}(k) f_{l \beta}(k),
$$

where the matrix $\mathbf{X}^{-1}$ is the inverse of $\mathbf{X} \equiv\left\{X_{\alpha \beta}\right\}$. The component $Q_{\gamma}^{\alpha \beta}(k)$ describes a partial contribution of the mode $Z_{\gamma}(k)$ to the time correlation function $f_{\alpha \beta}(k, t)$. Applying the Laplace transform to equation (26) yields the following result in the frequency space

$$
f_{\alpha \beta}^{\mathrm{M}}(k, \omega)=\sum_{\gamma=1}^{S} \frac{Q_{\gamma}^{\alpha \beta}(k)}{\mathrm{i} \omega+Z_{\gamma}(k)},
$$

which in the particular case of $\alpha=\beta=1$ can be considered as an alternative representation of extended continued fractions (13) for $g(k, \omega) \equiv f_{11}(k, \omega)$ in the Markovian approximation (18). The result (26) obtained in the $S$-mode description allows one to evaluate the $S \times S$ matrix of longitudinal and transverse TCFs (5).

From Eqs. (8) and (24) it can be easily shown that $\int_{0}^{\infty} \mathrm{d} t \mathbf{F}^{\mathrm{M}}(k, t)=\int_{0}^{\infty} \mathrm{d} t \mathbf{F}(k, t)$ or, in other words, $\lim _{\omega \rightarrow 0} \mathbf{F}^{\mathrm{M}}(k, \omega)=\lim _{\omega \rightarrow 0} \mathbf{F}(k, \omega)$ and the Markovian approximation leads directly to exact results in the low-frequency limit. Moreover, from the initial condition $\mathbf{F}^{\mathrm{M}}(k)=\mathbf{F}(k)$ (i.e., $\left.f_{\alpha \alpha}^{\mathrm{M}}(k)=f_{\alpha \alpha}(k), \alpha=0,1, \ldots, S-1\right)$ it follows that [34], if the $S$-mode approximation is used, time derivatives of the genuine $g(k, t)$ and approximated $g^{\mathrm{M}}(k, t)$ functions coincide at $t=0$ up to the $2(S-1)$-th order. For this reason, in a limit of $S \rightarrow \infty$ the Markovian approximation exactly reproduces analytical in time functions. Note, however, that due to the presence of "long-time tails" and other anomalies in dipolar systems, the time correlation functions $g(k, t)$ may be nonanalytic over a specific wavenumber range. In such a case, they are calculated only approximately, even if the hypothetical limit $S \rightarrow \infty$ is applied.

\section{Investigation of a Stockmayer fluid}

In order to verify the proposed theoretical scheme, we have considered the Stockmayer model of polar systems using the intermolecular potential $\varphi_{i j}=$ $\varphi_{\mathrm{LJ}}\left(r_{i j}\right)+\varphi_{\mathrm{dd}}\left(\boldsymbol{r}_{i j}, \boldsymbol{\mu}_{i}, \boldsymbol{\mu}_{j}\right)$, where $\varphi_{\mathrm{LJ}}\left(r_{i j}\right)=4 \epsilon_{\mathrm{LJ}}\left[\left(\sigma_{\mathrm{LJ}} / r_{i j}\right)^{12}-\left(\sigma_{\mathrm{LJ}} / r_{i j}\right)^{6}\right]$ denotes 
the Lennard-Jones (LJ) part, $\varphi_{\mathrm{dd}}=-3\left(\boldsymbol{\mu}_{i} \cdot \boldsymbol{r}_{i j}\right)\left(\boldsymbol{\mu}_{j} \cdot \boldsymbol{r}_{i j}\right) / r_{i j}^{5}+\left(\boldsymbol{\mu}_{i} \cdot \boldsymbol{\mu}_{j}\right) / r_{i j}^{3}$ describes the dipole-dipole interactions and $\boldsymbol{r}_{i j}=\boldsymbol{r}_{i}-\boldsymbol{r}_{j}$. Our numerical calculations were performed at the reduced density $n^{*}=\sigma_{\mathrm{LJ}}^{3} N / V=0.822$, mean temperature $T^{*}=$ $k_{\mathrm{B}} T / \epsilon_{\mathrm{LJ}}=1.147$, dipole moment $\mu^{* 2}=\mu^{2} /\left(\epsilon_{\mathrm{LJ}} \sigma_{\mathrm{LJ}}^{3}\right)=3.0$, the moment of inertia $J^{*}=J /\left(m \sigma_{\mathrm{LJ}}^{2}\right)=0.025$. The maximal order of extended continued fractions was restricted to $S=5$.

According to the basic results (13), (14), (21) and (22), the knowledge of static correlation functions $g_{2 s}(k)(15)$ is necessary at $s=0 \div 4$ to investigate the frequency dependence of the dielectric permittivity and evaluate the generalized dipolar mode spectra up to the five-variable description. There are several possibilities to define the static correlation functions. The lowest-order function $(s=0)$ presents the well-known Kirkwood factor $g(k)$ which is connected with the pair distribution function [29] and, therefore, can be calculated using one or another approach of the equilibrium statistical mechanics. The Kirkwood factor of the second order $(s=1)$ has an analytical representation $[26], g_{2}^{\mathrm{L}, \mathrm{T}}(k)=\frac{k_{\mathrm{B}} T}{3}\left(\frac{2}{J}+\frac{k^{2}}{m}\right)$. The higher-order functions $(s=2 \div 4)$ are related to the four-particle distribution function, and it is not a simple task to predict them theoretically. Usually, they are considered as adjustable parameters. It is obvious, however, that in such a way these functions cannot be determined uniquely. Because of this, to avoid any additional uncertainties in the calculation of collective modes and to observe the convergence of continued fractions in a pure form, we shall evaluate $g_{2 s}(k)$ using the molecular dynamics (MD) method. Details of our computer experiment are similar to those reported earlier [15].

We note that it is necessary to distinguish the correlation functions obtained directly in simulations for finite samples, $G_{s}(k)$, from infinite-system functions, $g_{s}(k)$. As it was shown previously, additional transformations to obtain $g(k)$ from $G(k)$ are necessary, namely, $g(k)=(1 / G(k)+D(k))^{-1}$ where $D(k)$ takes into account the details of simulations [15]. Moreover, the lowest-order time correlation functions related to infinite and finite systems obey the equality

$$
\frac{1}{g(k)-\mathrm{i} \omega \mathscr{L}_{\mathrm{i} \omega}(g(k, t))}=\frac{1}{G(k)-\mathrm{i} \omega \mathscr{L}_{\mathrm{i} \omega}(G(k, t))}+D(k) .
$$

Performing the Taylor expansion of (29) over inverse frequencies at $\omega \rightarrow \infty$, it can be easily shown that higher-order static correlation functions of the infinite system can be defined as follows:

$$
\begin{aligned}
& g_{2}(k)=G_{2}(k) \\
& g_{4}(k)=G_{4}(k)+D(k) G_{2}^{2}(k) \\
& g_{6}(k)=G_{6}(k)+D(k) G_{2}(k)\left(G_{4}(k)+g_{4}(k)\right) \\
& g_{8}(k)=G_{8}(k)+D(k)\left(G_{4}(k) g_{4}(k)+G_{2}(k)\left(G_{6}(k)+g_{6}(k)\right)\right) .
\end{aligned}
$$

The finite-system functions $G(k), G_{2}(k)$ and $G_{4}(k)$ were calculated directly in 
the simulations by the definition (15) using equation (1) and the explicit expressions

$$
\begin{aligned}
L \boldsymbol{M}(\boldsymbol{k})=\sum_{i=1}^{N} & {\left[\boldsymbol{w}_{i} \times \boldsymbol{\mu}_{i}-\mathrm{i} \boldsymbol{\mu}_{i}\left(\boldsymbol{k} \cdot \boldsymbol{v}_{i}\right)\right] \mathrm{e}^{-\mathrm{i} \boldsymbol{k} \cdot \boldsymbol{r}_{i}}, } \\
L^{2} \boldsymbol{M}(\boldsymbol{k})=\sum_{i=1}^{N}[ & {\left[\dot{\boldsymbol{w}}_{i} \times \boldsymbol{\mu}_{i}+\left(\left(\boldsymbol{w}_{i} \cdot \boldsymbol{\mu}_{i}\right) \boldsymbol{w}_{i}-\boldsymbol{w}_{i}^{2} \boldsymbol{\mu}_{i}\right)-\boldsymbol{\mu}_{i}\left(\boldsymbol{k} \cdot \boldsymbol{v}_{i}\right)^{2}\right.} \\
& \left.-\mathrm{i}\left(2 \boldsymbol{w}_{i} \times \boldsymbol{\mu}_{i}\left(\boldsymbol{k} \cdot \boldsymbol{v}_{i}\right)+\boldsymbol{\mu}_{i}\left(\boldsymbol{k} \cdot \dot{\boldsymbol{v}}_{i}\right)\right)\right] \mathrm{e}^{-\mathrm{i} \boldsymbol{k} \cdot \boldsymbol{r}_{i}},
\end{aligned}
$$

for higher-order dynamical variables, where $\dot{\boldsymbol{v}}_{i} \equiv L \boldsymbol{v}_{i}=-\frac{1}{m} \sum_{j(j \neq i)}^{N} \partial \varphi_{i j} / \partial \boldsymbol{r}_{i}$ $\equiv \frac{1}{m} \boldsymbol{f}_{i}$ and $\dot{\boldsymbol{w}}_{i} \equiv L \boldsymbol{w}_{i}=-\frac{1}{J} \sum_{j(j \neq i)}^{N} \boldsymbol{\mu}_{i} \times \partial \varphi_{i j} / \partial \boldsymbol{\mu}_{i} \equiv \frac{1}{J} \boldsymbol{K}_{i}$ denote the translational and rotational accelerations, respectively. In view of very complicated structures for $L^{3} \boldsymbol{M}(\boldsymbol{k})$ and $L^{4} \boldsymbol{M}(\boldsymbol{k})$, the highest-order static correlation functions $G_{6}(k)$ and $G_{8}(k)$ were evaluated numerically in terms of two- and four-fold time derivatives of the function $G_{4}(k, t)$ at $t=0$, i.e., $G_{6}(k)=-\partial^{2} G_{4}(k, t) /\left.\partial t^{2}\right|_{t=0}$ and $G_{8}(k)=\partial^{4} G_{4}(k, t) /\left.\partial t^{4}\right|_{t=0}$. The evaluation of $G_{6}(k)$ and $G_{8}(k)$ was carried out with the help of a special procedure to reduce numerical errors to a minimum.

The obtained in such a way longitudinal $g_{2 s}^{\mathrm{L}}(k)$ and transverse $g_{2 s}^{\mathrm{T}}(k)$ components of the infinite-system functions $g_{2 s}(k)$ (as well as $G_{2 s}^{\mathrm{L}, \mathrm{T}}(k)$ ) are displayed in fig. 1 at $s=0 \div 4$ in units of $\tau_{\sigma}^{-2 s}$, where $\tau_{\sigma}=\sigma_{\mathrm{LJ}}\left(\mathrm{m} / \epsilon_{\mathrm{LJ}}\right)^{1 / 2}$. The components $g_{2 s}^{\mathrm{L}, \mathrm{T}}(k)$, as autocorrelation static functions, are positively defined at arbitrary wavenumbers. In the limit of great wavevectors they can be calculated analytically, namely, $\lim _{k \rightarrow \infty} g_{2 s}^{\mathrm{L}, \mathrm{T}}(k)=(-1)^{s} \partial^{2 s} g_{\mathrm{G}}(k, t) /\left.\partial t^{2 s}\right|_{t \rightarrow 0}$, where $g_{\mathrm{G}}(k, t)=\frac{1}{3} \exp \left(-a k^{2} t^{2}\right)$ denotes the limiting Gaussian transition of $g^{\mathrm{L}, \mathrm{T}}(k, t)$ at $k \rightarrow \infty$ and $a=k_{\mathrm{B}} T / 2 m$ [26]. In particular, $g^{\mathrm{L}, \mathrm{T}}(k) \rightarrow \frac{1}{3}, g_{2}^{\mathrm{L}, \mathrm{T}}(k) \rightarrow \frac{2}{3} a k^{2}, g_{4}^{\mathrm{L}, \mathrm{T}}(k) \rightarrow 4 a^{2} k^{4}, g_{6}^{\mathrm{L}, \mathrm{T}}(k) \rightarrow 40 a^{3} k^{6}$ and $g_{8}^{\mathrm{L}, \mathrm{T}}(k) \rightarrow 560 a^{4} k^{8}$. It is interesting to note that higher-order functions differ from lower-order ones considerably. This indicates the existence of dynamical processes in the system which correspond to essentially different scales of time.

The results presented in figure 1 allow one to check immediately our assumption about the possibility of expressing the correlation time in terms of static correlation functions. The corresponding calculations (22) of the correlation time $\tau_{\text {cor }}^{(s)}(k)$ performed in different approximations $(s=1 \div 4)$, as well as the exact values (20) obtained by the MD method, are presented in figures $2 \mathrm{a}$ and $2 \mathrm{~b}$ for the cases of longitudinal and transverse fluctuations, respectively. As we can see from the figures, already the four-variable approximation reproduces the values of $\tau_{\text {cor }}(k)$ not only qualitatively, but even quantitatively over the whole region of wavenumbers.

The generalized dipolar mode spectra in two-, three-, four- and five-variable descriptions are shown in figure 3. In the case of transverse fluctuations (subsets (a) and (b) of the figure), we can clearly identify the diffusive mode $D_{1}(k)$ which is well separated from all the rest of the modes over a wide wavevector range. This mode 


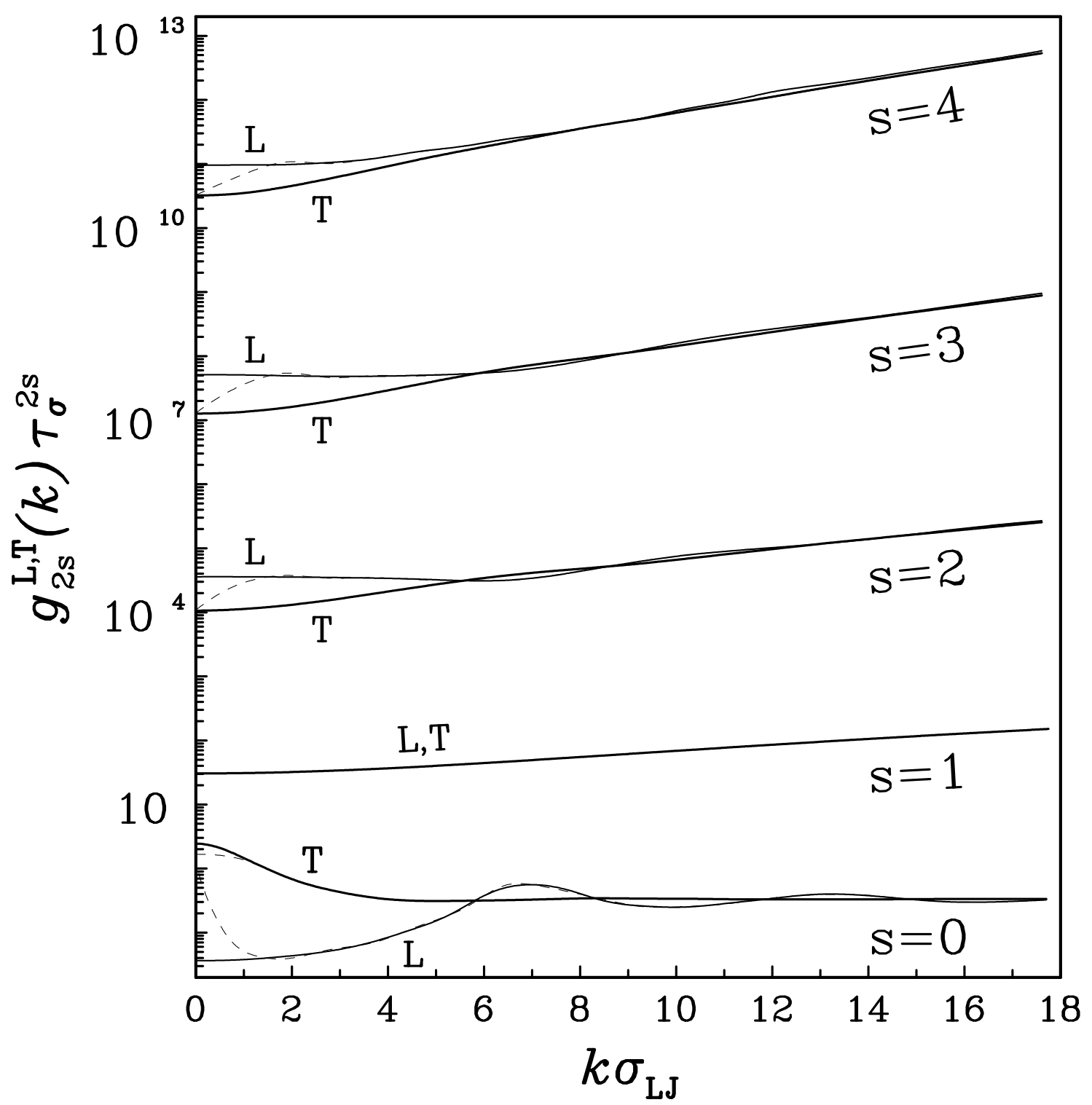

Figure 1. Transverse $(\mathrm{T})$ and longitudinal (L) components of the $s$-order static Kirkwood factors $(s=0 \div 4)$ for a Stockmayer fluid at $n^{*}=0.822$ and $T^{*}=1.147$. The MD data for the finite system are shown as dashed curves. The infinite-system Kirkwood factors are plotted by solid curves. Note that the transverse functions, corresponding to finite and infinite systems, are practically indistinguishable, excepting the case $s=0$. 

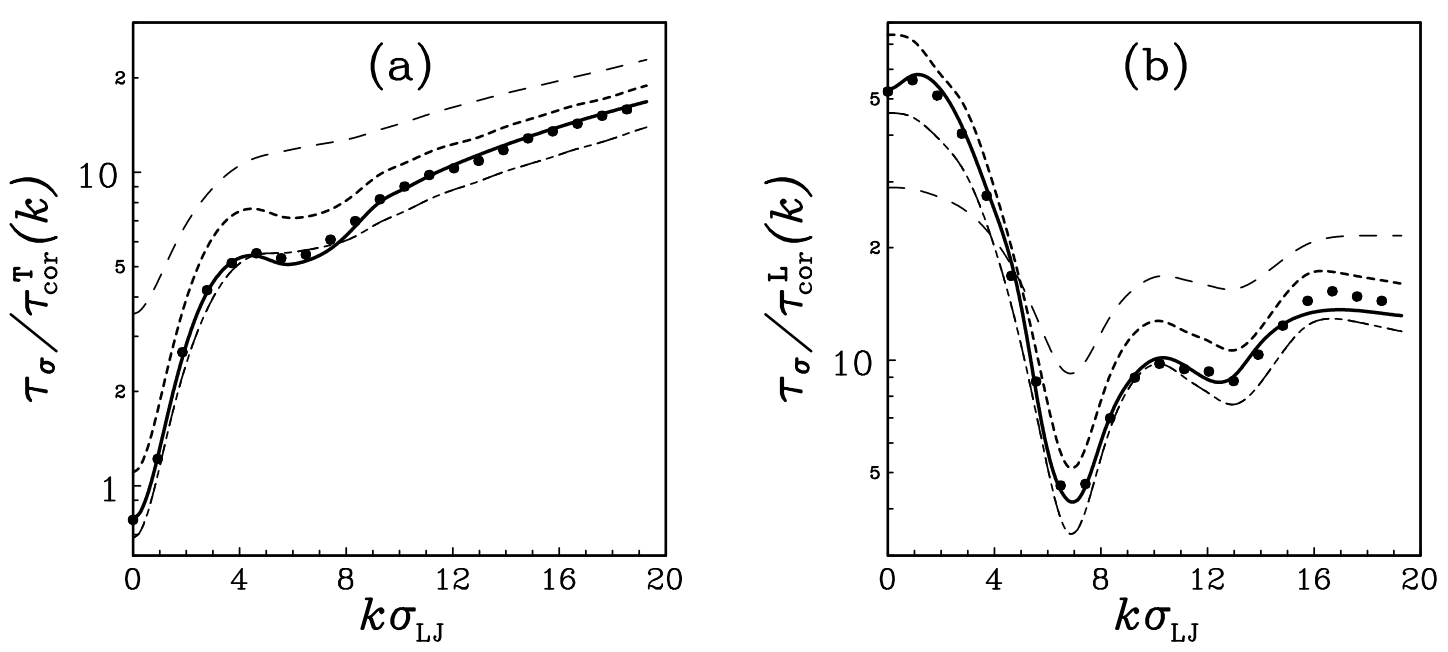

Figure 2. Transverse (a) and longitudinal (b) components of the correlation time for the Stockmayer fluid. The results in one-, two-, three- and four-order approximations are plotted by long-dashed, long-short-dashed, short-dashed and solid curves, respectively. The exact values are presented as circles.

converges rapidly to its genuine value with increasing the order of the approximation, so that it is reproduced quantitatively at small wavenumbers already within the two-variable description. As far as the five-variable description is used, we can talk about the quantitative reproducing at intermediate and great wavevectors as well. The appearance of the mode $D_{1}(k)$ is caused by the diffusive mechanism of dielectric relaxation in polar systems and only this mechanism is considered in the well-known Debye theory. Neglecting the dipole-dipole interactions, as it was done originally by Debye, one can find that in the infinite-wavelength limit $\lim _{k \rightarrow 0} D_{1}(k)=2 D_{\mathrm{R}}$, where $D_{\mathrm{R}}$ is a rotational diffusion coefficient. Applying the extended hydrodynamic approach, Bagchi and Chandra improved this result and obtained $D_{1}(k)=\left(2 D_{\mathrm{R}}+D_{\mathrm{T}} k^{2}\right)\left(1+\frac{N}{V} c(k)\right)$ where $D_{\mathrm{T}}$ is the translational diffusion coefficient and $c(k)$ denotes a component of the spherical harmonic expansion of the two-particle direct correlation function [4]. The latter result is valid not only for dilute systems but also for dense gases and liquids. However, it can be used at small wavevectors exclusively. Our scheme gives the possibility to define $D_{1}(k)$ in terms of the Kirkwood factor and its higher-order components at arbitrary values of wavenumber.

It is worth remarking that the diffusive mode, as the mode with the lowest real part, gives the main contribution to the TCFs and dielectric quantities in almost the whole domain of $k$-space, especially at small wavevectors. That is why the single-relaxation-time approximation for dipole-moment fluctuations, $\sim \mathrm{e}^{-D_{1}(k) t}$, which is used in the Debye theory, can be applied here. This approximation works well in the overdamped limit of large times $t$ and small frequencies $\omega$, where the inertial motions of the liquid molecules are not important. To describe properly the region of intermediate values of $t$ and $\omega$, it is necessary to consider higher-order modes. The next two propagating modes $P_{2}(k) \pm \mathrm{i} W_{2}(k)$ arise additionally begin- 

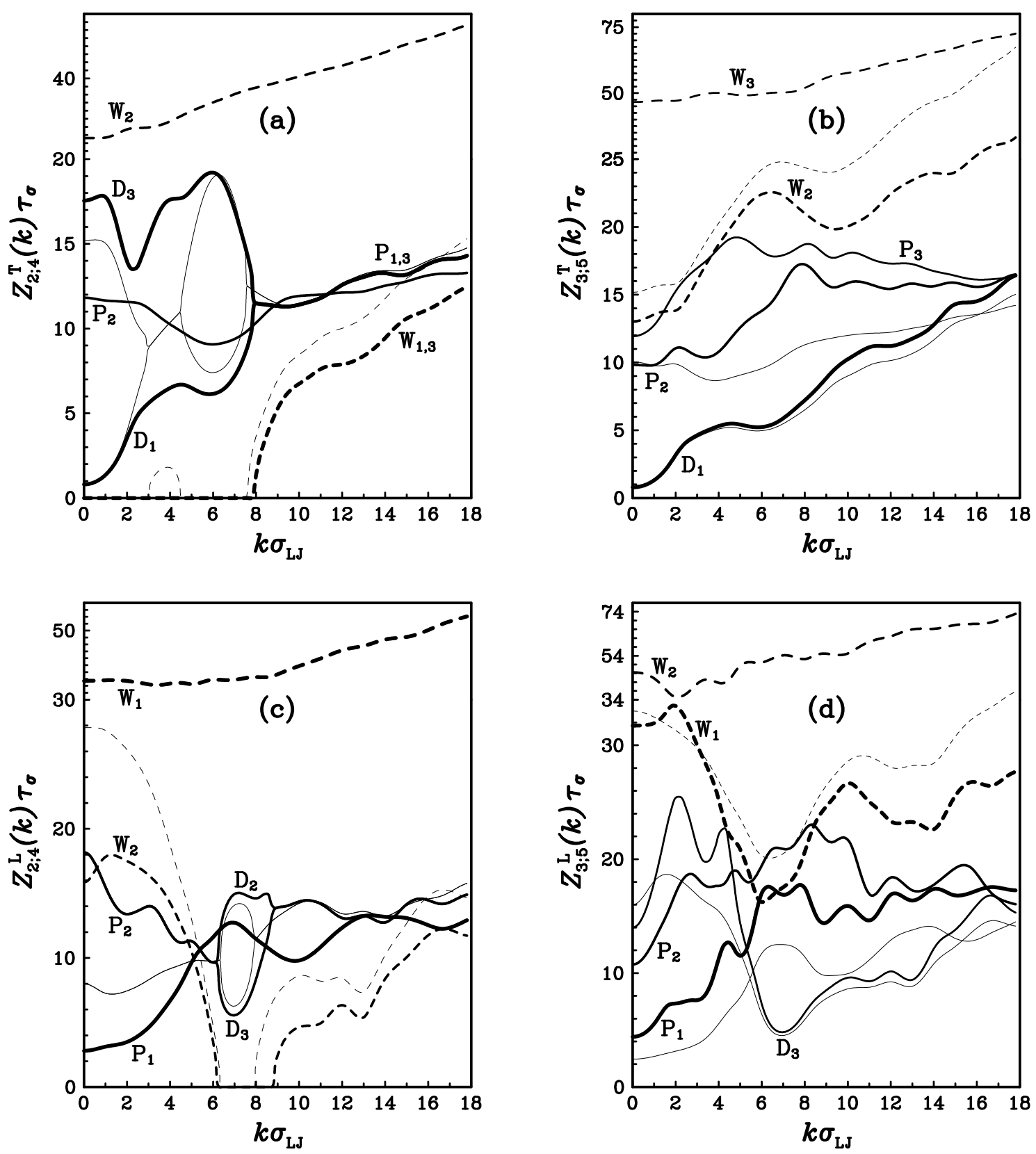

Figure 3. Generalized dipolar mode spectra of the Stockmayer fluid: transverse ((a), (b)) and longitudinal ((c), (d)) modes in four- ((a), (c)) and five- ((b), (d)) order descriptions. The pure diffusive modes, real and imaginary parts of propagating modes are marked by the symbols $\mathbf{D}, \mathbf{P}$ and $\mathbf{W}$, respectively. For the purpose of comparison the results in two- and three-mode approaches are shown in (a), (c) and (b), (d) by the thinnest curves. 
ning from the three-order approach. The three-, as well as higher-order descriptions include explicitly the free-motion effects in terms of $g_{2}(k)$ and correlations due to interactions via the torque-torque $\left\langle\sum_{i, j} \boldsymbol{K}_{i} \cdot \boldsymbol{K}_{j}\right\rangle$ and force-force $\left\langle\sum_{i, j} \boldsymbol{f}_{i} \cdot \boldsymbol{f}_{j}\right\rangle$ (at $k \neq 0$ ) fluctuations in terms of $g_{4}(k)$ (see Eq. (31)). Within the four-order approximation the secondary diffusive mode $D_{3}(k)$ appears at small $k$. It splits into two new propagating modes, $P_{3}(k) \pm \mathrm{i} W_{3}(k)$, with increasing the order of the approximation to five, whereas the previous two propagating modes are moderately corrected. The four- and five-mode descriptions consider higher-order kinetic processes which are important at very small times (very high frequencies) and large wavevector values. The transverse propagating modes describe an oscillation behaviour of time polarization fluctuations. However, it is hard to observe these oscillations because they damp significantly during their time periods, i.e., $P_{2}(k) \sim W_{2}(k)$ and $P_{3}(k) \sim W_{3}(k)$.

For the longitudinal fluctuations (subsets (c), (d) in fig. 3) the pattern is quite different. Here, we can easily distinguish two propagating modes, $P_{1}(k) \pm \mathrm{i} W_{1}(k)$. Contrary to the case of transverse fluctuations, these modes exhibit a quasiparticle feature at not very large wavenumbers, where $P_{1}(k) \ll W_{1}(k)$. They should be associated with dipolarons [30] (analogous to the well-known plasmons in Coulomb systems), where $W_{1}(k)$ and $P_{1}(k)$ define the frequency and damping of the dipolaron excitations, respectively. As one can see from the figures, the dipolaron mode is predicted already within the two-order description which includes the correct inertial short time behaviour of polarization fluctuations. If the dipole-dipole interactions are neglected, the dipolaron frequency can be defined approximately as $\lim _{k \rightarrow 0} W_{1}(k)=\frac{4 \pi N \mu^{2}}{V J} \approx 35.2 \tau_{\sigma}^{-1}$ that is very close to the values $27.8,33.0,35.5$ and $31.7 \tau_{\sigma}^{-1}$ obtained by us at $S=2,3,4$ and 5 , respectively. The secondary oscillation process is reproduced by the next two complex-conjugated modes $P_{2}(k) \pm \mathrm{i} W_{2}(k)$ at $S=4$. Finally, in the five-order description the pure diffusive mode $D_{3}(k)$ appears additionally.

The inequalities $P_{1}(k) \lesssim W_{1}(k), P_{2}(k), D_{3}(k)$ can be considered as a condition of existing the dipolaron oscillations. This condition is satisfied as far as $k^{*} \equiv k \sigma_{\mathrm{LJ}} \lesssim 4$. With increasing wavevector values the diffusive processes begin to dominate, especially at $k^{*} \sim 7$, where $D_{3}(k)$ is much less than all the other modes. This feature is visible in all the orders of the approximations as well. For example, in the fourorder description the propagating modes $P_{2}(k) \pm \mathrm{i} W_{2}(k)$ are separated into the two pure diffusive modes $D_{2}(k)$ and $D_{3}(k)$ within a small region near $k^{*} \sim 7$, where the longitudinal component $g^{\mathrm{L}}(k)$ of the Kirkwood factor has a sharp maximum (see figure 1).

In view of the behaviour of dipolar modes, the whole region of wavevectors can be split into several characteristic intervals. In the first one the lowest-lying dipolar modes are well separated from the rest of the modes and this separation is observed as long as $k^{*} \lesssim 2$ (the so-called extended hydrodynamic regime). In this interval the Debye-like theory can be applied to transverse dipole-moment fluctuations, whereas the longitudinal component of the dielectric permittivity can be predicted by two complex-conjugated dipolaron modes. In the second range of intermediate 
wavenumbers, $2 \lesssim k^{*} \lesssim 12$, all the modes are mixed in a very complicated manner (especially in the case of longitudinal fluctuations). To describe the dynamical behaviour of dielectric quantities in this range, involving additional higher-order modes is necessary, excepting the subinterval $6 \lesssim k^{*} \lesssim 8$, where the longitudinal diffusive mode dominates over all the rest of the modes, similar to the behaviour of transverse modes in the extended hydrodynamic regime. Finally, in the so-called free-motion regime, $k^{*} \gtrsim 12$, all the modes tend to their own linear asymptotes to reproduce the Gaussian time shape $g_{\mathrm{G}}(k, t)$ of dipole-moment fluctuations.

Examples of the normalized time correlation functions $\Phi_{\mathrm{L}, \mathrm{T}}(k, t) \equiv g^{\mathrm{L}, \mathrm{T}}(k, t) /$ $g^{\mathrm{L}, \mathrm{T}}(k)$ obtained in two-, three- and five-mode descriptions are presented and compared with the MD data [15] in figure 4. As we can see from the figure, the transverse component $\Phi_{\mathrm{T}}(k, t)$ exhibits an almost pure damped feature over a wide range of wavenumbers. In the case of longitudinal fluctuations this is valid for intermediate and great wavevector values only. At small wavevectors the longitudinal dipole-moment fluctuations are described by strong dipolaron oscillations with a slight damping. Such a behaviour of $\Phi_{\mathrm{L}, \mathrm{T}}(k, t)$ is completely in line with the predictions of the generalized dipolar modes approach. The transverse TCFs are reproduced satisfactorily even within the three-mode description. The longitudinal oscillations are described in this case as well, but only qualitatively. At the same time, the approximated and genuine TCFs begin to be indistinguishable with increasing the order of the description to five.

A similar pattern to that presented for TCFs is observed for the wavevectorand frequency-dependent dielectric permittivity $\varepsilon_{\mathrm{L}, \mathrm{T}}(k, \omega)=\varepsilon_{\mathrm{L}, \mathrm{T}}^{\prime}(k, \omega)-\mathrm{i} \varepsilon_{\mathrm{L}, \mathrm{T}}^{\prime \prime}(k, \omega)$. Our calculations, carried out in one-, two-, three- and five-order approximations for the longitudinal $\varepsilon_{\mathrm{L}}(k, \omega)$ and transverse $\varepsilon_{\mathrm{T}}(k, \omega)$ components are shown in figures 5 and 6 , respectively, in comparison with the MD data of paper [15]. We note that in the infinite-wavelength $\operatorname{limit}_{k \rightarrow 0} \varepsilon_{\mathrm{L}, \mathrm{T}}(k, \omega)=\varepsilon(\omega)$. It can be easily seen that within the Debye-like theory $(S=1)$ the dielectric permittivity can be well reproduced in the hydrodynamic limit (low frequencies and wavenumbers). With increasing wavevector and frequency values this theory fails especially in the case of longitudinal fluctuations. In the three-mode approximation we can talk about a qualitative description. Finally, within the five-variable approach the entire frequency dependence of the dielectric permittivity is described quantitatively at arbitrary wavevectors (deviations from the MD data do not exceed a few per cent). This merely means that the five variables constitute an almost complete set of slow quantities and the Markovian approximation begins to be almost exact. Therefore, the extended continued fractions (13) converge rapidly with increasing the order of the approximation and the hypothesis of an abbreviated description is in excellent accord.

\section{Conclusion}

It has been established that dielectric relaxation in a dipolar fluid can be successfully studied within the generalized mode method. The proposed approach can 

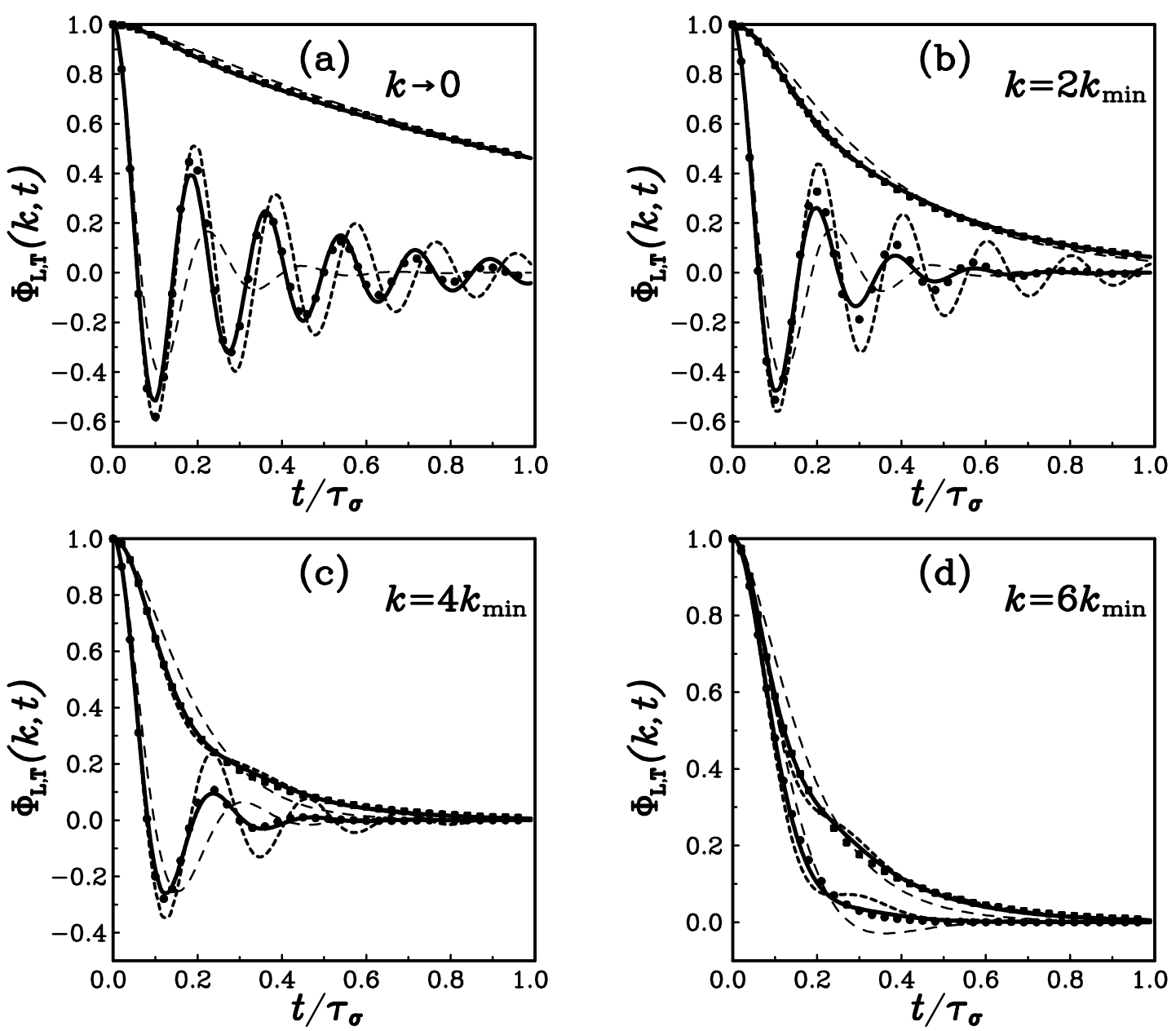

Figure 4. The normalized time autocorrelation functions of the dipole-moment fluctuations for the Stockmayer fluid at some fixed values of wavenumber, where $k_{\min }=2 \pi / V^{1 / 3}=0.927 / \sigma_{\mathrm{LJ}}$. The $\mathrm{MD}$ data for longitudinal and transverse components are shown as circles and squares. The results of two-, three- and five-mode descriptions are plotted by the corresponding long-, short-dashed and solid curves, respectively. 

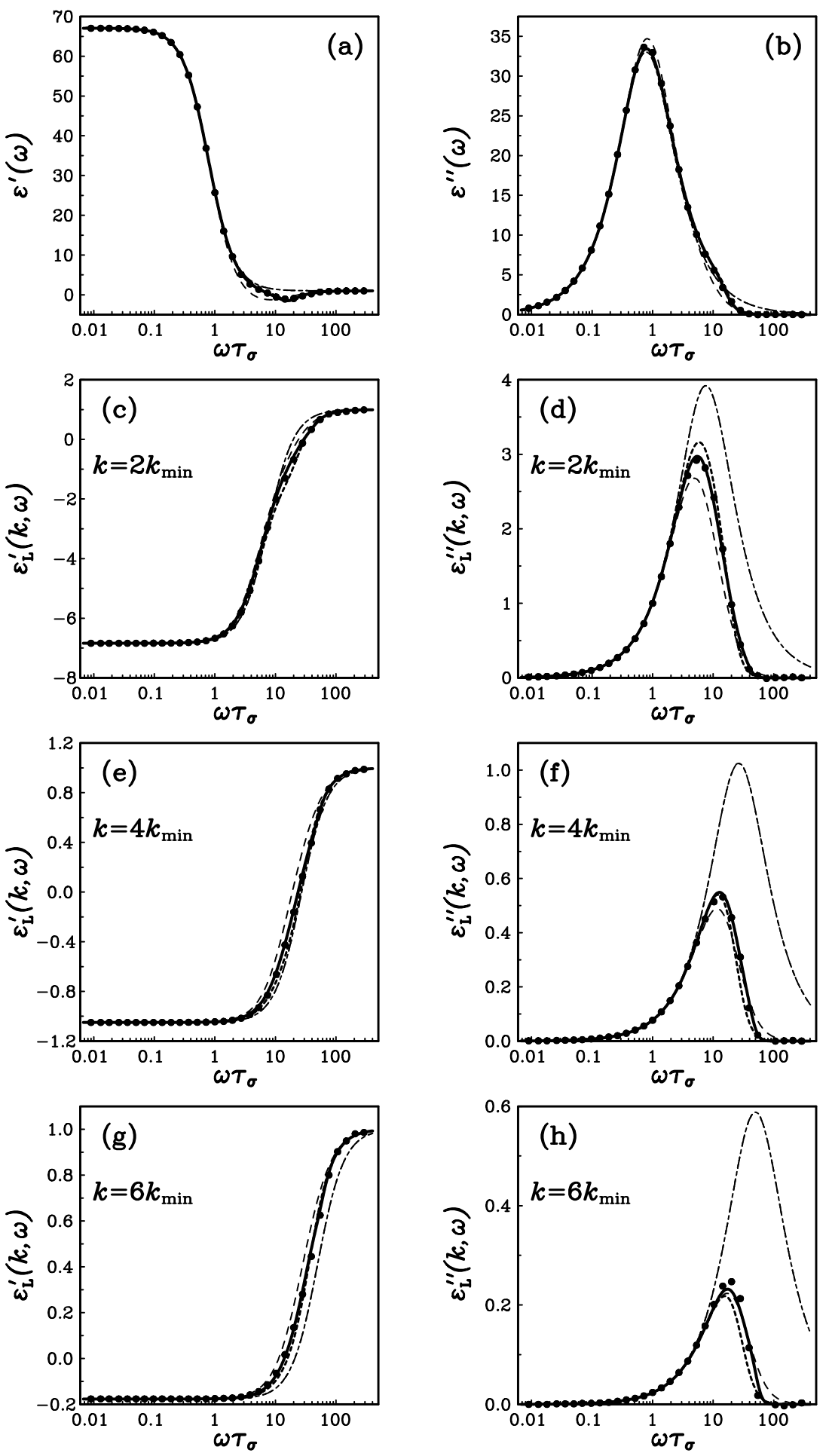

Figure 5. The frequency-dependence of the longitudinal dielectric permittivity for the Stockmayer fluid at infinite ((a), (b)) and finite ((c)-(h)) wavelengths. The MD data are shown by circles. The results obtained within one-, two-, threeand five-mode descriptions are plotted by long-short-, long-, short-dashed and solid curves, respectively. 

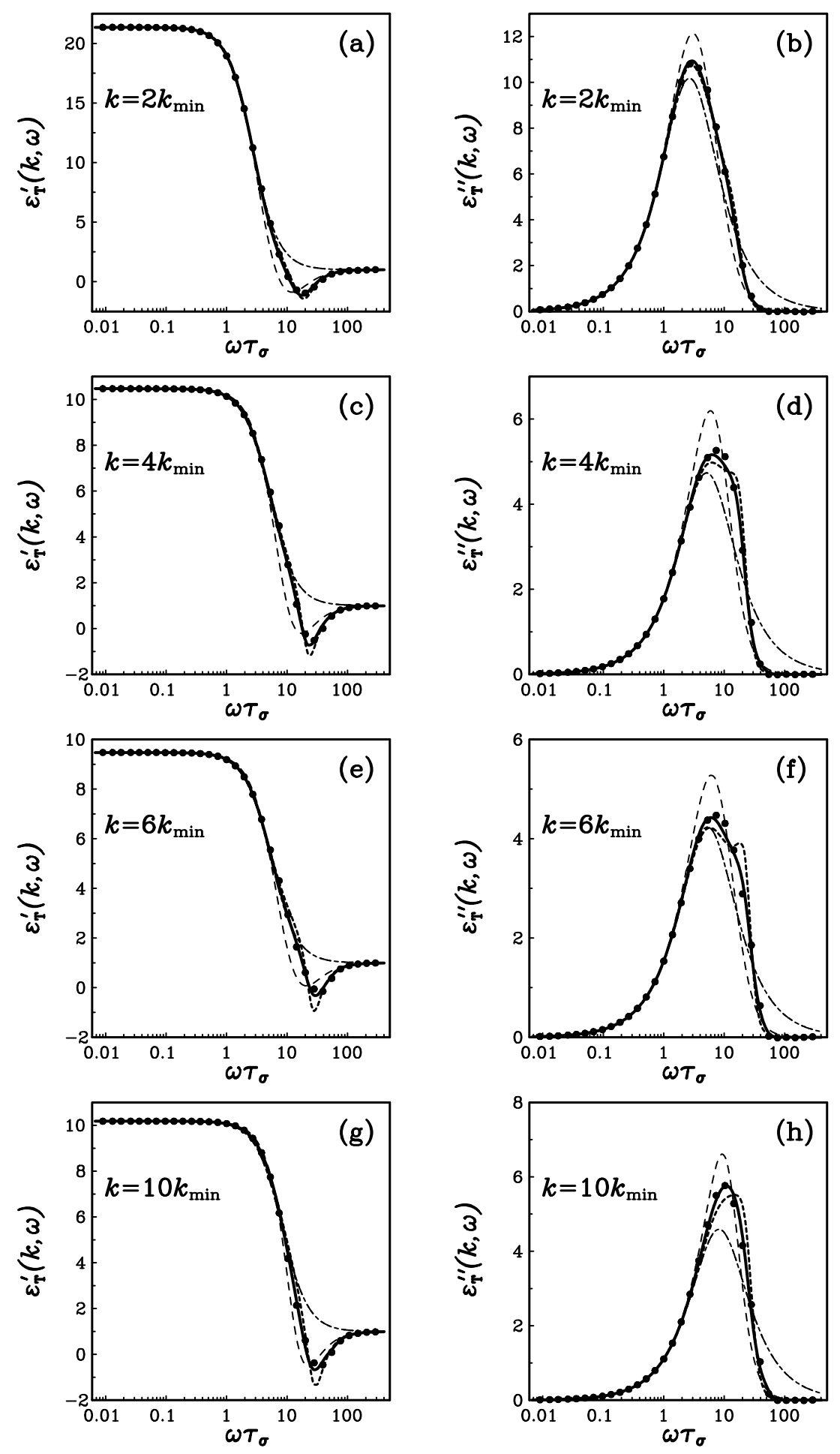

Figure 6. The frequency-dependence of the transverse dielectric permittivity for the Stockmayer fluid at finite wavelengths. Notations are as for fig. 5 . 
be considered as an extension of the three-variable theory of Madden and Kivelson [2] to arbitrary numbers of dynamical variables. Our scheme for the computation of dipolar modes is presented in such a form that is very convenient for actual applications. In particular, all the necessary input quantities are, in fact, static Kirkwood factors of different orders. The static factors can be determined by either equilibrium theories or direct computer simulations. This has allowed us both to avoid any fitting procedures and to evaluate the generalized dipolar-mode spectra of a Stockmayer fluid over the whole scale of wavelengths within up to the five-order description for the first time. It has been shown on the basis of direct calculations that the five-variable theory enables one to define quantitatively the dielectric permittivity of a dipolar fluid at arbitrary wavevector and frequency values.

The investigation of generalized hydrodynamic modes of a Stockmayer fluid, as well as the application of the proposed theory to more complicated interaction site models of polar fluids will we presented in separate publications.

\section{Acknowledgements}

One of us (I.P.) would like to acknowledge financial support of the President of Ukraine. I.M. also thanks the "Fonds für Förderung der wissenschaftlichen Forschung" under Project P12422TPH for financial support.

\section{References}

1. Kivelson D., Keyes T. Unified theory of orientational relaxation. // J. Chem. Phys., 1972, vol. 57, No 11, p. 4599-4612.

2. Madden P., Kivelson D. A consistent molecular treatment of dielectric phenomena. // Adv. Chem. Phys., 1984, vol. 56, p. 467-566.

3. Chandra A., Bagchi B. Microscopic expression for frequency and vector dependent dielectric constant of a dipolar liquid. // J. Chem. Phys., 1989, vol. 90, No 3, p. 1832-1840.

4. Bagchi B., Chandra A. Collective orientational relaxation in dense dipolar liquids. // Adv. Chem. Phys., 1991, vol. 80, p. 1-126.

5. Kim S.H., Vignale G., DeFacio B. Frequency- and wave-vector-dependent dielectric function of waterlike fluids. // Phys. Rev. A, 1992, vol. 46, No 12, p. 7548-7560.

6. Dávalos-Orozco L.A., del Castillo L.F. Dielectric relaxation in polar and viscoelastic fluids with internal rotation. // J. Chem. Phys., 1992, vol. 96, No 12, p. 9102-9113.

7. Chandra A., Wei D., Patey G.N. Dielectric relaxation of dipolar liquids. // J. Chem. Phys., 1993, vol. 99, No 3, p. 2068-2073.

8. Impey R.W., Madden P.A., McDonald I.R. Spectroscopic and trasport properties of water. Model calculations and the interpritation of experimental results. // Mol. Phys., 1982, vol. 46, No 3, p. 513-539.

9. Edwards D.M.F., Madden P.A., McDonald I.R. A computer simulation study of the dielectric properties of a methil cyanide. I. The rigid dipole case. // Mol. Phys., 1984, vol. 51, No 5, p. 1141-1161. 
10. Neumann M., Steinhauser O., Pawley G.S. Consistent calculation of the static and frequency-dependent dielectric constant in computer simulations. // Mol. Phys., 1984, vol. 52, No 1, p. 97-113.

11. Neumann M. The dielectric constant of water. Computer simulations with the MCY potential. // J. Chem. Phys., 1985, vol. 82, No 12, p. 5663-5672.

12. Neumann M. Dielectric relaxation of water. Computer simulations with TIP4P potential. // J. Chem. Phys., 1986, vol. 85, No 3, p. 1567-1580.

13. Bertolini D., Tani A. Generalized hydrodynamics and the acoustic modes of water: Theory and simulation results. // Phys. Rev. E, 1995, vol. 51, No 2, p. 1091-1118.

14. Omelyan I.P. Temperature behavior of the frequency-dependent dielectric constant for a Stockmayer fluid. // Phys. Lett. A, 1996, vol. 216, p. 211-216.

15. Omelyan I.P. Wavevector- and frequency-dependent dielectric constant of the Stockmayer fluid. // Mol. Phys., 1996, vol. 87, No 6, p. 1273-1283.

16. Hasted J.B. Aqueous dielectrics. Chapman and Hall, London, 1973.

17. Berne B., Pecora R. Dynamic light scattering. John Wiley, New York, 1976.

18. Fleming G.R. Chemical applications of ultrafast spectroscopy. Oxford University Press, Oxford, 1986.

19. Hansen J.P., McDonald I.R. Theory of Simple Liquids. Academic Press, New York, 1986.

20. Omelyan I.P., Zhelem R.I., Tokarchuk M.V. Generalized hydrodynamics of polar liquids in an external inhomogeneous electric field. The NSO method. // Ukr. Fiz. Zh., 1997, vol. 42, No 6, p. 684-692 (in Ukrainian).

21. Mryglod I.M., Omelyan I.P., Tokarchuk M.V. Generalized collective modes for the Lennard-Jones fluid. // Mol. Phys., 1995, vol. 84, No 2, p. 235-259.

22. Mryglod I.M., Omelyan I.P. Generalized collective modes for a Lennard-Jones fluid in higher-mode approximations. // Phys. Lett. A, 1995, vol. 205, p. 401-406.

23. Mryglod I.M., Omelyan I.P. Generalized mode approach: 1. Transverse time correlation functions and generalized shear viscosity of a Lennard-Jones fluid. // Mol. Phys., 1997, vol. 90, No 1, p. 91-99.

24. Mryglod I.M., Omelyan I.P. Generalized mode approach: 2. Longitudinal time correlation functions of a Lennard-Jones fluid. // Mol. Phys., 1997, vol.91, No 6, p. 1005-1015.

25. Mryglod I.M., Omelyan I.P. Generalized mode approach: 3. Generalized transport coefficients of a Lennard-Jones fluid. // Mol. Phys., 1997 (in press).

26. Omelyan I.P. Generalized collective mode approach in the dielectric theory of dipolar systems. // Physica A, 1997, vol. 247, No 1/4, p. 121-139.

27. Mori H. Transport, collective motion and Brownian motion. // Prog. Theor. Phys., 1965, vol. 33, No 3, p. 423-455.

28. Mori H. A continued-fraction representation of the time-correlation functions. // Prog. Theor. Phys., 1965, vol. 34, No 3, p. 765-776.

29. Neumann M. Computer simulation and the dielectric constant at finite wavelength. // Mol. Phys., 1986, vol. 57, No 1, p. 97-121.

30. Pollock E.L., Alder B.J. Frequency-dependent dielectric response in polar liquids. // Phys. Rev. Lett., 1981, vol. 46, No 14, p. 950-953. 


\title{
Діелектрична релаксація у дипольних рідинах. Підхід узагальнених мод
}

\author{
І.П.Омелян, І.М.Мриглод, М.В.Токарчук \\ Інститут фізики конденсованих систем НАН України, \\ 290011 м. Львів, вул. Свєнціцького 1 \\ Отримано 28 листопада 1997 р.
}

Концепція узагальнених колективних мод, яка недавно була запропонована для дослідження простих рідин, застосовується зараз до опису процесів діелектричної релаксації в дипольних системах. Даний підхід представляє собою розширення формалізму дипольної густини до довільного числа динамічних змінних і значень довжин хвиль. Спектри узагальнених дипольних мод Штокмайєрівської рідини визначено в широкій області довжин хвиль у наближеннях аж до п'ятого порядку. Діелектрична сприйнятливість, залежна від хвильового вектора і частоти, та часова автокореляційна функція дипольного моменту обчислюються на основі аналітичних виразів, використовуючи дипольні моди. Отримані результати порівнюються з результатами нижчепорядкових наближень і даними молекулярної динаміки. Показано, що п'яти-модовий опис кількісно відтворює діелектричну функцію в усій області зміни хвильового вектора і частоти.

Ключові слова: колективні моди, діелектричні властивості, узагальнена гідродинаміка

PACS: $67.55 . J d ; 77.22 .-d ; 47.65$ 